\title{
Delivery of nutrition services in health systems in sub-Saharan Africa: opportunities in Burkina Faso, Mozambique and Niger
}

\author{
Rachel D Hampshire ${ }^{1, *}$, Victor M Aguayo ${ }^{2}$, Hamani Harouna ${ }^{3}$, Julie A Roley ${ }^{4}$, Ann Tarini ${ }^{1}$ \\ and Shawn K Baker ${ }^{5}$ \\ ${ }^{1}$ Helen Keller International, BP 8150 Ouagadougou 04, Burkina Faso: ${ }^{2}$ UNICEF Regional Office for West and \\ Central Africa, BP 29720, Dakar, Senegal: ${ }^{3} \mathrm{HKI} /$ Niger: ${ }^{4} \mathrm{HKI} /$ Mozambique: ${ }^{5} \mathrm{HKI}$ Africa Region
}

Submitted 18 August 2003: Accepted 21 May 2004

\begin{abstract}
Background: In sub-Saharan Africa, underweight and micronutrient deficiencies account for an estimated $25 \%$ of the burden of disease. As the coverage of national health systems expands, increased opportunities exist to address the needs of children and women, the most vulnerable to these deficiencies, through high-quality nutrition services.

Objectives: To assess health providers' knowledge and practice with regard to essential nutrition services for women and children in Burkina Faso, Mozambique and Niger, in order to assist the development of a standard guide and tools to assess and monitor the quality of the nutrition services delivered through national health systems.

Findings: The three surveys reveal the extent of missed opportunities to deliver nutrition services during routine prenatal, postnatal and child-care consultations for the prevention and treatment of highly prevalent nutritional deficiencies.

Conclusion: A commitment to improving the quality of facility-based nutrition services is necessary to impact on the health outcomes of women and children 'covered' by national health systems. Rigorous assessment and monitoring of the quality of nutrition services should inform health programme and policy development. Building on the lessons learned in these three assessments, Helen Keller International has developed a standard Guide and Tools to assess the quality of the nutrition services delivered through national health systems. These tools can be adapted to assess ongoing nutrition services in health facilities, provide a framework for nutrition programming, inform the development of pre-service as well as inservice nutrition training curricula for providers, and evaluate the impact of nutrition training on providers' practices.
\end{abstract}

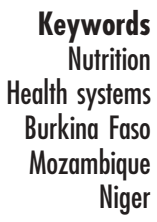

Recent evidence shows that underweight and micronutrient deficiencies (vitamin A, iron and zinc) account for an estimated $15 \%$ of the global burden of disease and represent the leading risk factors of disease, disability and death world-wide ${ }^{1}$. Women and young children are the most vulnerable to these nutritional deficiencies. In the developing world, more than 150 million children are underweight and 450 million women of reproductive age are stunted (height $<145 \mathrm{~cm})^{1}$; an estimated 140 million children of pre-school age and more than 7 million pregnant women are vitamin-A-deficient ${ }^{2} ; 48 \%$ of infants and young children ( $0-23$ months), $42 \%$ of women of reproductive age and over $50 \%$ of pregnant women are anaemic $^{3}$; and an estimated 2 billion people live in countries at risk of iodine deficiency ${ }^{4}$. Recent analyses show that two-thirds of child deaths are associated with nutritional deficiencies ${ }^{5}$, and there is increasing evidence that micronutrient deficiencies, such as vitamin A deficiency, explain a significant proportion of the burden of maternal morbidity and mortality ${ }^{6}$. In sub-Saharan Africa, underweight, vitamin A, iron and zinc deficiencies account for close to $25 \%$ of the disease burden ${ }^{1}$.

Nutrition services provided through national health systems offer an incomparable opportunity to address some of the nutrition needs facing women and young children, if providers know, understand and adhere to national protocols. In sub-Saharan Africa, governments and their development partners work to expand the proportion of the total population covered by health services. However, studies show that the practice of delivering the nutrition services outlined in national health protocols varies greatly within and among countries. In Botswana for example, 69\% of mothers attending consultations reported having received counselling on exclusive breast-feeding from their health providers ${ }^{7}$, whereas in Togo 63\% of mothers did not ${ }^{8}$. In this sense, 
the quality of nutrition services directed at mothers is lacking.

Expanding the reach of health services alone is therefore not enough to ensure that vulnerable populations will receive the nutrition services supported by national governments. Monitoring the quality of the nutrition services offered to women and young children can strengthen the ability of health systems to provide services that their clients perceive as valuable and thus seek out. This is a precondition for the effectiveness and impact of facility-based nutrition services.

Between September 2000 and September 2001, Helen Keller International (HKI) assessed the quality of the nutrition services offered through national health systems in three sub-Saharan African countries, namely Burkina Faso, Mozambique and Niger, by exploring three main areas - staff, facility and service factors - in an effort to inform policy and programme development.

\section{Objectives and methods}

A shared objective of the three studies was to assess health providers' knowledge and practice of the essential nutrition services included in the norms and procedures of the national ministries of health regarding pregnant women (prenatal consultations), women in the early postpartum period (postnatal consultations) and children under 5 years of age (well-child and sick-child consultations). The objective of this review was to develop, building on the lessons learned in Burkina Faso, Mozambique and Niger, a standard guide and tools to assess the quality of the nutrition services delivered through national health systems. This paper presents some of the most relevant findings from the three assessments and illustrates the most common challenges and opportunities that health systems encounter in the provision of nutrition services in subSaharan Africa. A comparison among the three study areas is not one of the authors' objectives.
A survey team (three to six interviewers per team) conducted the quality assessment in each of the three countries. A supervisor co-ordinated the work of each survey team. Four types of survey tools were used to collect the information: a provider questionnaire, a consultation observation checklist, a client exit interview questionnaire and a facility checklist. When possible, all providers at observed facilities were interviewed. An effort was made to gather client interviews from both clients who were observed during their consultation as well as from clients who received services without an observer present, in an effort to minimise the bias created by having an observer present during consultation.

The sample of facilities included in each assessment was chosen following country-specific criteria to build a purposive sample that would maximise representativeness (rural-urban, public-private, large-small) while minimising logistical and financial constraints. In Niger, the assessment was conducted in the districts of Maine Soroa, N'Guigmi and Diffa in the department of Diffa, eastern Niger. A total of 15 health facilities (five facilities per district) were included in the assessment. In Burkina Faso, the assessment was conducted in the districts of Pissy in central Burkina Faso and Fada in the south-east. A total of 18 facilities (eight in Pissy and 10 in Fada) were included in the assessment. In Mozambique, the assessment was conducted in Manica Province, west-central Mozambique. A total of 10 facilities were included in the assessment (Table 1). The specific details of each country assessment are reported elsewhere ${ }^{9-11}$.

\section{Findings}

Findings from the three country assessments are limited to examinations of provider knowledge of national protocols and the practices concerning those norms. Differences between practices observed by the research teams and reported by clients are noted. Adherence to specific

Table 1 Types of facilities surveyed in Niger, Burkina Faso and Mozambique

\begin{tabular}{|c|c|c|c|}
\hline & Name of facility & Characteristics of facility & $\begin{array}{l}\text { Number } \\
\text { assessed }\end{array}$ \\
\hline \multirow[t]{3}{*}{ Burkina Faso } & District Medical Centre & A district health facility that has surgery capabilities & 4 \\
\hline & Medical Centre & A health facility that may include a doctor & 1 \\
\hline & Health Promotion Centre & $\begin{array}{l}\text { The most basic health facility in the national system, } \\
\text { staffed by nurse(s) }\end{array}$ & 13 \\
\hline \multirow[t]{2}{*}{ Mozambique } & Health Centre & $\begin{array}{l}\text { Peri-urban to rural town facility. } \\
\text { Those in district capitals may include a doctor on staff }\end{array}$ & 6 \\
\hline & Health Post & $\begin{array}{l}\text { Rural town or remote rural facility. } \\
\text { Staff usually includes } 1 \text { or } 2 \text { providers: nurses, } \\
\text { a health worker and/or a midwife }\end{array}$ & 4 \\
\hline \multirow[t]{3}{*}{ Niger } & District Hospital & $\begin{array}{l}\text { Maternal health-care services available and staff } \\
\text { includes a trained midwife for care }\end{array}$ & 3 \\
\hline & Integrated Health Centre Type II & $\begin{array}{l}\text { Staffed by two agents, both nurses, plus a third } \\
\text { health agent and maternal health-care facilities }\end{array}$ & 3 \\
\hline & Integrated Health Centre Type I & Staffed by two agents, both nurses & 9 \\
\hline
\end{tabular}


dosage regimens and time period appropriateness of services were not examined.

\section{Nutrition care for women during pregnancy (Table 2)}

\section{Iron deficiency and anaemia}

Prenatal screening for clinical signs of anaemia and counselling on iron deficiency/anaemia control were the nutrition services most consistently offered to pregnant women in the three countries. In Niger, 95\% of pregnant women observed at the prenatal consultation were examined for conjunctive pallor (a clinical sign of anaemia) and $73 \%$ were asked about recent symptoms of anaemia. In Niger, daily supplementation with iron and folic acid, beginning at first prenatal contact, is a policy of the Ministry of Health. Health providers' knowledge reflects this, as $97 \%$ of those interviewed $(n=30)$ reported that they systematically prescribed iron + folic acid supplements to pregnant women. Practice differed from knowledge, however, as only $57 \%$ of women observed at prenatal consultation received a prescription or counselling for the procurement of iron + folic acid supplements despite the fact that iron + folic acid supplements were available in $73 \%$ of the health facilities included in the survey. Seventy-five per cent of the women interviewed after their prenatal consultation reported that they had received a prescription for iron + folic acid supplements, but only $32 \%$ of the women who received them were able to explain that the supplements were to prevent/control iron deficiency or anaemia. In Mozambique, only 26\% of women observed at prenatal consultation were screened for clinical signs of anaemia (pallor in hands and inner eyelids). Sixty-six per cent of all women both observed during and interviewed following prenatal consultation received a prescription for iron + folic acid supplements. In Burkina Faso, all women observed at prenatal consultation were examined for clinical signs of anaemia. Sixty-seven per cent of women observed during and 97\% of women interviewed after prenatal consultation had received a prescription for iron + folic acid supplements.
Forty-two per cent were able to give reasons such as 'to have more blood' or 'to have more strength' as benefits of taking the prescribed supplements.

\section{Malaria}

In Niger, malaria prophylaxis with chloroquine for pregnant women is a policy of the Ministry of Health. All health providers interviewed reported that they systematically prescribed chloroquine for malaria prophylaxis to pregnant women. However, only $60 \%$ of women observed at prenatal consultation were prescribed chloroquine as a prophylactic measure for malaria. Of the pregnant women interviewed after prenatal consultation, $72 \%$ had received a prescription for chloroquine and $89 \%$ of those who received a prescription knew that it was 'to avoid malaria'. Chloroquine was available in 93\% of the health centres included in the survey. In Burkina Faso, 64\% of women observed at prenatal consultation were given a prescription for chloroquine but only $16 \%$ of the women interviewed after prenatal consultation had received counselling on malaria prophylaxis with chloroquine despite the Ministry of Health policy in this sense. In Mozambique, malaria prophylaxis with chloroquine for pregnant women is not a policy of the Ministry of Health. Only $11 \%$ of the women interviewed after prenatal consultation had received a prescription for treatment of malaria with chloroquine.

\section{Vitamin A and iodine deficiencies}

In Niger, only $22 \%$ of pregnant women observed during prenatal consultation were asked whether they had or were experiencing episodes of night blindness (a clinical sign of vitamin A deficiency). This percentage was even lower in Mozambique (3\%) and Burkina Faso, where none of the women observed during prenatal consultation were asked about pregnancy-specific visual disorders. In Burkina Faso, only $4 \%$ of the health providers interviewed $(n=24)$ reported looking for evidence of goitre (a clinical sign of iodine deficiency) in the physical examination of women attending prenatal consultations. In Mozambique, none of

Table 2 Nutrition services for pregnant women surveyed in Niger, Burkina Faso and Mozambique: prenatal consultations*

\begin{tabular}{|c|c|c|c|c|c|c|}
\hline & \multicolumn{2}{|c|}{ Niger (September 2000) } & \multicolumn{2}{|c|}{$\begin{array}{l}\text { Burkina Faso (August/ } \\
\text { September 2001) }\end{array}$} & \multicolumn{2}{|c|}{$\begin{array}{c}\text { Mozambique } \\
\text { (September 2001) }\end{array}$} \\
\hline & $\begin{array}{l}\text { Interviews } \\
(n=88)\end{array}$ & $\begin{array}{l}\text { Observations } \\
\quad(n=37)\end{array}$ & $\begin{array}{l}\text { Interviews } \\
(n=159)\end{array}$ & $\begin{array}{l}\text { Observations } \\
\quad(n=56)\end{array}$ & $\begin{array}{l}\text { Interviews } \\
(n=129)\end{array}$ & $\begin{array}{l}\text { Observations } \\
\quad(n=73)\end{array}$ \\
\hline $\begin{array}{l}\text { Screening for clinical signs of anaemia } \\
\text { (palm and/or eye pallor) }\end{array}$ & & $\mathrm{X}$ & & $\mathrm{X}$ & $\mathrm{X}$ & $\mathrm{X}$ \\
\hline Exploration of symptoms of anaemia & & $\mathrm{X}$ & & & $\mathrm{X}$ & $X$ \\
\hline Prescription of iron + folic acid supplements & $\mathrm{X}$ & $X$ & $\mathrm{X}$ & $\mathrm{X}$ & $X$ & $\mathrm{X}$ \\
\hline Prescription of malaria prophylaxis & $X$ & $\mathrm{X}$ & $\mathrm{X}$ & $X$ & $\mathrm{X}$ & $\mathrm{X}$ \\
\hline Exploration of clinical signs of vitamin A deficiency & & $X$ & & $x$ & $\mathrm{X}$ & $\mathrm{X}$ \\
\hline Screening for clinical signs of iodine deficiency & & & & & $\mathrm{X}$ & $\mathrm{X}$ \\
\hline $\begin{array}{l}\text { Exploration of dietary habits and counselling } \\
\text { on dietary improvement }\end{array}$ & $\mathrm{X}$ & $\mathrm{X}$ & $\mathrm{X}$ & $x$ & $X$ & $X$ \\
\hline
\end{tabular}

* Services addressed for each target group in each country reflect the established norms and protocols of each country's Ministry of Health, unless specifically noted otherwise in the text. 
the women observed during prenatal consultation were examined for clinical signs of iodine deficiency and only $5 \%$ of the women interviewed after prenatal consultation reported having been examined for signs of goitre.

\section{Dietary habits/improvement}

In Mozambique, 69\% of women observed during prenatal consultation were asked about their dietary habits during pregnancy and 59\% were counselled on dietary improvement during pregnancy. In Niger and Burkina Faso, only $38 \%$ and $14 \%$ respectively of the women observed at prenatal consultation received counselling on pregnancyrelated dietary improvement. The women interviewed after their prenatal consultation mirrored the low emphasis placed on dietary and nutritional counselling observed at prenatal consultations. In Niger, only 3\% of the women interviewed reported that they had been questioned regarding their dietary habits, while 11\% had received some form of nutritional counselling. In Burkina Faso, 14\% of women interviewed stated they had received nutritional counselling in the course of their prenatal consultation. Women's energy balance can be improved by increasing energy intake and/or decreasing energy expenditure. In Niger, $80 \%$ of the providers interviewed reported that counselling women to increase their periods of rest during pregnancy was important advice for prenatal consultation. Similarly, $75 \%$ of the health providers interviewed in Burkina Faso reported that increased rest was part of regular counselling given to pregnant women during prenatal consultations; however, only $11 \%$ of Burkinabé women interviewed following prenatal consultation had been advised to rest more during pregnancy.

\section{Nutrition care for women in the early postnatal period (Table 3)}

\section{Iron deficiency and anaemia}

The proportion of new mothers who were examined for clinical signs of anaemia and/or asked about anaemia-related symptoms varied significantly among the three countries. In Niger, $62 \%$ of women observed at postnatal consultations were examined for clinical signs of anaemia (pallor) and were asked about symptoms of anaemia. Ninety-five per cent of the women observed and $85 \%$ of those interviewed after postnatal consultation were prescribed iron + folic acid supplements. In Burkina Faso, $62 \%$ of women observed at postnatal consultation were examined for clinical signs of anaemia and asked about symptoms of anaemia. Thirty-one per cent of the women observed and $32 \%$ of the women interviewed were advised to continue taking iron + folic acid supplements. In Mozambique, only 18\% of women observed at postnatal consultation were examined for clinical signs of anaemia and only $20 \%$ of those observed during or interviewed after postnatal consultation were advised to continue taking iron + folic acid supplements to treat signs of anaemia. National policy for 3 months of preventive postpartum iron + folic acid supplementation has since been established in Mozambique.

\section{Malaria}

In Niger, $47 \%$ of the women observed and $58 \%$ of those interviewed were advised to take or continue taking malaria prophylaxis, while this proportion was a mere $6 \%$ in Mozambique among the women observed at postnatal consultation. As with prenatal care, chloroquine is prescribed in Mozambique as a malaria treatment, not as a prophylaxis. In Burkina Faso, $40 \%$ of the health providers interviewed $(n=20)$ reported that they regularly prescribed a malaria prophylaxis to new mothers.

\section{Vitamin A and iodine deficiencies}

Providers' concern for postnatal vitamin A deficiency was poor in all three countries. In Niger, $38 \%$ of the postnatal consultations observed included questions regarding clinical signs of vitamin A deficiency such as night blindness. Thirty-eight per cent of the women observed and $17 \%$ of those interviewed received a vitamin A supplement; however, only $22 \%$ of the women interviewed

Table 3 Nutrition services for postpartum women surveyed in Niger, Burkina Faso and Mozambique: postnatal consultations

\begin{tabular}{|c|c|c|c|c|c|c|}
\hline & \multicolumn{2}{|c|}{ Niger (September 2000) } & \multicolumn{2}{|c|}{$\begin{array}{l}\text { Burkina Faso (August/ } \\
\text { September 2001) }\end{array}$} & \multicolumn{2}{|c|}{$\begin{array}{c}\text { Mozambique } \\
\text { (September 2001) }\end{array}$} \\
\hline & $\begin{array}{l}\text { Interviews } \\
(n=52)\end{array}$ & $\begin{array}{l}\text { Observations } \\
\quad(n=21)\end{array}$ & $\begin{array}{l}\text { Interviews } \\
(n=27)\end{array}$ & $\begin{array}{l}\text { Observations } \\
\quad(n=13)\end{array}$ & $\begin{array}{l}\text { Interviews } \\
(n=50)\end{array}$ & $\begin{array}{l}\text { Observations } \\
\quad(n=39)\end{array}$ \\
\hline $\begin{array}{l}\text { Screening for clinical signs of anaemia } \\
\text { (palm and/or eye pallor) }\end{array}$ & & $\mathrm{X}$ & & $\mathrm{X}$ & $\mathrm{X}$ & $\mathrm{X}$ \\
\hline Exploration of symptoms of anaemia & & $X$ & & $\mathrm{X}$ & $X$ & $\mathrm{X}$ \\
\hline Prescription of iron + folic acid supplements & $\mathrm{X}$ & $X$ & $\mathrm{X}$ & $X$ & $X$ & $\mathrm{X}$ \\
\hline Prescription of malaria prophylaxis & $\mathrm{x}$ & $\mathrm{X}$ & & & $\mathrm{X}$ & $\mathrm{x}$ \\
\hline Exploration of clinical signs of vitamin A deficiency & & $X$ & & $\mathrm{X}$ & $X$ & $\mathrm{X}$ \\
\hline Vitamin A supplementation & $\mathrm{X}$ & $X$ & $\mathrm{X}$ & $X$ & & \\
\hline Counselling for potential iodine deficiency & & & & & $x$ & $\mathrm{X}$ \\
\hline $\begin{array}{l}\text { Exploration of dietary habits and counselling } \\
\text { on dietary improvement }\end{array}$ & $\mathrm{X}$ & $\mathrm{X}$ & $\mathrm{x}$ & $\mathrm{x}$ & $\mathrm{x}$ & $\mathrm{x}$ \\
\hline Counselling on early initiation of breast-feeding & $\mathrm{X}$ & & $\mathrm{X}$ & & & \\
\hline Counselling on exclusive breast-feeding & $\mathrm{X}$ & & $x$ & $\mathrm{X}$ & $\mathrm{X}$ & $\mathrm{X}$ \\
\hline
\end{tabular}


were able to explain that it was 'for good health' or 'to prevent night blindness' ('dundumi'). In Burkina Faso, none of the women observed was examined for/asked about clinical signs of vitamin A deficiency. None of the women observed and only 15\% of those interviewed after postnatal consultation received either a vitamin A supplement or a prescription for it, despite the existing national policy in this sense. In Mozambique, only 3\% of health providers observed at postnatal consultation asked women whether they were experiencing episodes of night blindness and only $2 \%$ of women interviewed reported that they had been examined for clinical signs of iodine deficiency (goitre).

\section{Dietary habits/improvement}

Counselling on dietary improvement during the postnatal period also varied significantly across countries. In Niger, $62 \%$ of women observed at postnatal consultations were asked about their appetite and $17 \%$ of the women interviewed following postnatal consultation reported that they had been recommended to increase their intake of vitamin-A-rich foods, specifically liver, fish and sauces with green leafy vegetables. In Burkina Faso, 15\% of women observed at postnatal consultations were asked about their dietary habits and $15 \%$ of women interviewed after their consultation were able to remember a suggestion for dietary improvement. In Mozambique, $82 \%$ of the women observed at postnatal consultations and $92 \%$ of those interviewed were asked about their appetite and diet.

\section{Nutrition care for young children (Table 4)}

\section{Breast-feeding}

In Niger, $89 \%$ of the health providers interviewed $(n=29)$ reported that they regularly counselled mothers to exclusively breast-feed infants younger than 6 months. However, although $46 \%$ of the new mothers interviewed reported that their health providers had counselled them regarding early initiation of breast-feeding, only $6 \%$ were advised to breast-feed their infant exclusively for the first 6 months of life. In Mozambique, $87 \%$ of the new mothers observed during consultations received counselling on breast-feeding but only $8 \%$ were advised to breastfeed their infant exclusively for 4 months (in accordance with the policy of the Ministry of Health). In Burkina Faso, $80 \%$ of health providers believed that exclusive breastfeeding for 6 months is feasible. Among those who did not, the two major reasons cited were the potential dehydration of the infant and the inability of health providers to convince mothers that breast milk provides all an infant needs during the first 6 months of life. Fifty-six per cent of the new mothers interviewed in Burkina Faso reported having received counselling on the early initiation of breast-feeding and 52\% reported having been counselled to breast-feed exclusively for the first 6 months. Only $48 \%$ of the women interviewed believed that they would be able to successfully feed their infants only breast milk until 6 months of age. Among the women who believed that exclusive breast-feeding was not feasible, the reasons most frequently given were that they had never heard of it being done or had never heard of anyone in their family giving only breast milk to their infants.

\section{Growth promotion}

Child weighing was routinely performed in all three countries included in the assessments. However, results were rarely used to guide subsequent nutritional counselling tailored to the individual child. In Niger, for example, all of the children observed at well-child consultations were weighed but only $62 \%$ of their caregivers were asked and/or counselled about the feeding/dietary habits of the child. Similarly, only $70 \%$ of the caregivers interviewed after the consultation reported that the provider gave them advice pertaining to the diet of the child. In the case of sick children, only $64 \%$ of those observed were weighed and $24 \%$ of their caregivers were questioned/counselled on feeding/dietary habits. In Burkina Faso, all children observed but one were weighed while only 29\% of caregivers were asked/counselled about the feeding/dietary habits of the child. In Mozambique, $72 \%$ of the children observed were weighed but the percentage of

Table 4 Nutrition services for children 0-59 months old surveyed in Niger, Burkina Faso and Mozambique: well-child and sick-child consultations

\begin{tabular}{|c|c|c|c|c|c|c|}
\hline & \multicolumn{2}{|c|}{ Niger (September 2000) } & \multicolumn{2}{|c|}{$\begin{array}{l}\text { Burkina Faso (August/ } \\
\text { September 2001) }\end{array}$} & \multicolumn{2}{|c|}{$\begin{array}{c}\text { Mozambique } \\
\text { (September 2001) }\end{array}$} \\
\hline & $\begin{array}{l}\text { Interviews } \\
(n=224)\end{array}$ & $\begin{array}{l}\text { Observations } \\
\quad(n=91)\end{array}$ & $\begin{array}{l}\text { Interviews } \\
(n=280)\end{array}$ & $\begin{array}{l}\text { Observations } \\
(n=100)\end{array}$ & $\begin{array}{l}\text { Interviews } \\
(n=147)\end{array}$ & $\begin{array}{l}\text { Observations } \\
\quad(n=78)\end{array}$ \\
\hline Weighing of the child & $\mathrm{X}$ & $\mathrm{X}$ & $\mathrm{X}$ & $\mathrm{X}$ & $\mathrm{x}$ & $\mathrm{X}$ \\
\hline $\begin{array}{l}\text { Counselling on complementary feeding/ } \\
\text { dietary improvement }\end{array}$ & $x$ & $x$ & & $x$ & $x$ & $x$ \\
\hline $\begin{array}{l}\text { Screening for clinical signs of anaemia } \\
\text { (palm and/or eye pallor) }\end{array}$ & & $x$ & $x$ & $\mathrm{x}$ & $x$ & $x$ \\
\hline $\begin{array}{l}\text { Exploration of signs and symptoms } \\
\text { of vitamin A deficiency }\end{array}$ & & $x$ & & $\mathrm{x}$ & $\mathrm{x}$ & $x$ \\
\hline Vitamin A supplementation & $\mathrm{X}$ & $\mathrm{x}$ & $\mathrm{X}$ & $\mathrm{x}$ & $\mathrm{x}$ & $\mathrm{X}$ \\
\hline
\end{tabular}


caregivers asked about the child's appetite and feeding habits was only $41 \%$ and $26 \%$, respectively. Only $12 \%$ of caregivers interviewed after the consultation reported having been given advice on issues related to feeding/ dietary habits.

\section{Micronutrient deficiencies}

In Niger, the date of the child's last vitamin A dose was verified in only $23 \%$ of the consultations observed. Twenty-two per cent of eligible children at well-child consultations received a vitamin A supplement whereas the percentage of eligible sick children who did so was only $12 \%$. Thirteen per cent of caregivers interviewed reported that their child had received a vitamin A capsule during the consultation but only $37 \%$ of the caregivers whose child received a vitamin A capsule were able to explain why their child had received it. In Burkina Faso, none of the providers observed looked for clinical signs of vitamin A deficiency; 19\% of the caregivers interviewed reported that their child had received a vitamin A capsule but only $4 \%$ of the children observed during consultation received one. In Mozambique, $6 \%$ of the child consultations observed included investigation of night blindness as a clinical sign of vitamin A deficiency and $7 \%$ of the caregivers interviewed acknowledged that their child had received a vitamin A supplement. Screening for clinical signs of anaemia in children was rare in all three countries. The percentage of child consultations observed where children were screened for clinical signs of anaemia was only $13 \%, 12 \%$ and $4 \%$ in Niger, Burkina Faso and Mozambique, respectively.

\section{Discussion}

Women who seek facility-based prenatal care offer providers with an opportunity to address the specific nutritional needs of the pregnant woman and the growing foetus. Postnatal consultations give health professionals the occasion to provide new mothers with the nutritional care and support they need to address the nutritional stress associated with pregnancy and lactation; they also give providers a unique opportunity to ensure adequate nutritional care for the newborn. Well-child contacts (particularly growth monitoring and promotion and immunisation consultations) allow providers to establish or reinforce positive practices and prevent or address early growth faltering and nutritional deficiencies, including micronutrient deficiencies, particularly among infants and young children ( $0-23$ months). Sick-child consultations (especially those related to measles, malaria, acute respiratory infections and diarrhoeal diseases) are additional key contact opportunities between providers and caregivers, particularly as a growing number of countries such as Burkina Faso, Mozambique and Niger implement and expand the Integrated Management of
Childhood Illnesses, which includes nutrition aspects of the care of sick children.

Mariko explains that two components of 'quality of care' exist - that of 'perceived quality' (by the clients being served) as well as 'normative quality' or adherence to a certain standard ${ }^{12}$. This idea of expressing measures of quality from two different points of view is echoed by Boller et al. in their assessment of public and private health facilities in Tanzania ${ }^{13}$. Rigorous assessment and monitoring of the quality of nutrition services provided by national health systems should incorporate both components of 'quality' when used to inform programme and policy development. In all three cases examined here, the quality of services provided by national health systems left significant room for improved delivery of essential nutrition services for the prevention and treatment of highly prevalent nutritional deficiencies among women and children. As the coverage of health care systems improves in developing countries, so must the delivery of key nutrition services provided to the population groups at higher risk of nutritional deficiencies be improved and maintained. Governments and their development partners must therefore ensure that the nutrition services provided by their national health systems are of the highest quality possible.

Using different survey tools and approaches, the three assessments illustrate well the extent of opportunities often missed during routine prenatal, postnatal and child-care consultations for improving the nutritional status of the women and children 'covered' by national health-care systems. Building on the lessons learned in Burkina Faso, Mozambique and Niger, HKI has developed a standard Guide and Tools for Quality Assessment of Nutrition Services in Health Systems (http://www.hkiafrica.org/Guide_and_Tools.html)

designed to ascertain the quality of nutrition services provided through national health systems by addressing both clients and providers. The Tools included in the Guide can be adapted to:

- assess ongoing nutrition services in health facilities;

- provide a framework to facility-based nutrition programming for governments and their development partners;

- inform the development of both pre-service and inservice nutrition training curricula for health providers; and

- evaluate the impact of nutrition training on providers' practices.

The objective of the Guide and Tools is to assist national health systems in improving the nutrition services they deliver as coverage of these services expands. The improved quality will stimulate demand and utilisation of these services and prevent further missed opportunities to impact positively on the health outcomes of the most vulnerable populations. 


\section{Acknowledgements}

This paper is a product of HKI with support from the Canadian International Development Agency (CIDA) through The Micronutrient Initiative (MI). Additional technical inputs were provided with support from the United States Agency for International Development (USAID). The opinions expressed in this paper are those of the authors and do not necessarily reflect the views of CIDA, MI or USAID.

\section{References}

1 World Health Organization (WHO). The World Health Report, 2002. Reducing Risks, Promoting Healthy Life. Geneva: WHO, 2003.

2 West KP Jr. Extent of vitamin A deficiency among preschool children and women of reproductive age. Journal of Nutrition 2002; 132: 2857S-66S.

3 The Micronutrient Initiative (MI). Iron Improves Life. Ottawa: MI, 2003.

4 World Health Organization (WHO)/United Nations Children's Fund/International Council for the Control of Iodine Deficiency Disorders. Progress Towards the Elimination of Iodine Deficiency Disorders. Geneva: WHO, 1999.

5 Fishman S, Caulfield LE, de Onis M, Blösnner M, Hyder AA, Mullany L, et al. Childhood and maternal underweight. In: Ezzati M, Lopez AD, Rodgers A, Murray CJL, eds. Comparative Quantification of Health Risks: Global and Regional Burden of Disease Attributable to Selected Major Risk Factors. Geneva: World Health Organization, 2003.
6 West KP Jr, Katz J, Khatry SK, LeClerq SC, Pradhan EK, Shrestha SR, et al. Double blind, cluster randomized trial of low dose supplementation with vitamin A or beta carotene on mortality related to pregnancy in Nepal. The NNIPS-2 Study Group. British Medical Journal 1999; 318: 570-5.

7 Aguayo VM, Ross JS, Kanon S, Ouedraogo AN. Monitoring compliance with the International Code of Marketing of Breastmilk Substitutes in west Africa: multisite cross sectional survey in Togo and Burkina Faso. British Medical Journal 2003; 326: 381-3.

8 Mahgoub Salah EO, Bandeke T, Nnyepi M. Breastfeeding in Botswana: practices, attitudes, patterns and the sociocultural factors affecting them. Journal of Tropical Pediatrics 2002; 48: 195-9.

9 Helen Keller International (HKI). Enquête sur la qualité des préstations de la prise en charge nutritionnelle des enfants de moins de cinq ans et des femmes enceintes et en postpartum dans le Département de Diffa au Niger. Niamey, Niger: HKI, 2001.

10 Helen Keller International (HKI). Enquête sur la prise en charge nutritionnelle dans les formations sanitaires de Burkina Faso. Ouagadougou, Burkina Faso: HKI, 2001.

11 Helen Keller International (HKI). Health Facility Assessment: Nutrition and Micronutrients in Manica Province, Mozambique. Maputo, Mozambique: HKI, 2002.

12 Mariko M. Quality of care and the demand for services in Bamako, Mali: the specific roles of structural, process and outcome components. Social Science and Medicine 2003; 56: $1183-96$.

13 Boller C, Wyss K, Mtasiwa D, Tanner M. Quality and comparison of antenatal care in public and private providers in the United Republic of Tanzania. Bulletin of the World Health Organization 2003; 81: 116-22. 